Pacific Journal of Mathematics

AUTOMORPHISMS OF LOCALLY COMPACT GROUPS 


\title{
AUTOMORPHISMS OF LOCALLY COMPACT GROUPS
}

Justin Peters ANd TerJe Sund

\begin{abstract}
It is proved that for arbitrary locally compact groups $G$ the automorphism group $\operatorname{Aut}(G)$ is a complete topological group. Several conditions equivalent to closedness of the group Int $(G)$ of inner automorphisms are given, such as $G$ admits no nontrivial central sequences. It is shown that Aut $(G)$ is topologically embedded in the automorphism group Aut $\mathscr{R}(G)$ of the group von Neumann algebra. However, closedness of Int $\mathscr{R}(G)$ does not imply closedness of $\operatorname{Int}(G)$, nor conversely.
\end{abstract}

1. Let $G$ be a locally compact group and Aut $(G)$ the group of all its topological automorphisms with the Birkhoff topology. A neighborhood basis of the identity automorphism consists of sets $N(C, V)=\left\{\alpha \in \operatorname{Aut}(G): \alpha(x) \in V x\right.$ and $\alpha^{-1}(x) \in V x$, all $\left.x \in C\right\}$, where $C$ is compact and $V$ is a neighborhood of the identity $e$ of $G$. As is well known, Aut $(G)$ is a Hausdorff topological group but not generally locally compact $[1 ; \mathrm{p} .57]$. In this article we are mainly concerned with the topological properties of Aut $(G)$ and its subgroup Int $(G)$ of inner automorphisms. We prove that for $G$ arbitrary locally compact Aut $(G)$ is a complete topological group. In particular, if $G$ is also separable Aut $(G)$ is a Polish group. Furthermore, we give two new characterizations of the topology for Aut $(G),(1.1$ and 1.6). In $\S 2$ we turn to the question of when certain subgroups (among them Int $(G)$ ) are closed in Aut $(G)$, and several equivalent conditions are given; for instance, Int $(G)$ is closed iff $G$ admits no nontrivial central sequences (2.2). Applications to more special classes of groups are also given, as well as to the question of unimodularity of $\operatorname{Int}(G)$, (2.7). We remark that there is no separability assumption on the groups before 1.11 .

LEMMA 1.1. The sets

$$
W_{\phi_{1}, \ldots, \phi_{n} ; \varepsilon}=\left\{\tau \in \operatorname{Aut}(G) ;\left\|\phi_{j}^{\circ} \tau-\phi_{j}\right\|_{\infty}<\varepsilon, 1 \leqq j \leqq n\right\}
$$

where $\phi_{j} \in C_{o}(G)$ and $\varepsilon>0$, form a basis for the neighborhoods of the identity in Aut $(G)$.

Proof. Let $\phi_{1}, \cdots, \phi_{n} \in C_{c}(G)$ and $\varepsilon>0$ be given. Note that $\left\|\phi_{j} \circ \tau-\phi_{j}\right\|_{\infty}<\varepsilon$ implies $\left\|\phi_{j} \circ \tau^{-1}-\phi_{j}\right\|_{\infty}<\varepsilon, \tau \in \operatorname{Aut}(G)$. Set $F=\bigcup_{i=1}^{n}$ support $\left(\phi_{i}\right)$, and let $W$ be a symmetric neighborhood of $e$ in $G$ such that $\left|\phi_{i}(x)-\phi_{i}(w x)\right|<\varepsilon$ for all $x \in G, w \in W, 1 \leqq i \leqq n$. We claim 
$N(F, W) \leqq W_{\phi_{1}, \ldots, \phi_{n} ; \varepsilon^{\circ}}$ Let $\tau \in N(F, W)$. Then for $x \in F, \tau(x) x^{-1} \in W$, so $(*)$

$$
\left|\phi_{i}(x)-\phi_{i}(\tau(x))\right|<\varepsilon, \quad 1 \leqq i \leqq n .
$$

If $\tau(x) \in F$, then $\tau^{-1}(\tau(x)) \tau(x)^{-1} \in W$, i.e., $x \tau(x)^{-1} \in W$, so (*) holds. If $x \notin F$ and $\tau(x) \notin F$ then $\phi_{i}(x)=\phi_{i}(\tau(x))=0$, so again $(*)$ is satisfied.

Conversely, let $F \subset G$ be compact and $W$ a neighborhood of $e$ in $G$. Let $U$ be a compact neighborhood of $e$ in $G$ such that $U^{2} \cdot U^{-1} \subset$ $W$. Let $\psi \in C_{c}(G)$ be such that $0 \leqq \psi \leqq 1$, support $(\psi) \subset U^{2}$, and $\psi(u) \geqq 1 / 2 \forall u \in U$. (The existence of such a $\psi$ is clear.) Let $\left\{x_{1}, \cdots, x_{n}\right\}$ be a finite subset of $F$ such that $\left\{U_{x_{i}}: 1 \leqq i \leqq n\right\}$ covers $F$. Define $\psi_{i} \in C_{c}(G)$ by $\psi_{i}(x)=\psi\left(x x_{i}^{-1}\right), 1 \leqq i \leqq n$. It is now routine to verify that $W_{\psi_{1}, \ldots, \psi_{n}: 1 / 2} \subset N(F, W)$.

1.2. By Braconnier [1] there is a continuous (modular) homomorphism $\Delta: \operatorname{Aut}(G) \rightarrow R^{+}$with the property

$$
\Delta(\alpha)^{-1} \int_{G} f \circ \alpha^{-1}(x) d x=\int_{G} f(x) d x, \quad \text { for } f \in C_{c}(G),
$$

where $d x$ is a fixed Haar measure. Defining

$$
\tilde{\alpha}(f)=\Delta(\alpha)^{-1} f \circ \alpha^{-1}, f \in L^{1}(G), \alpha \in \operatorname{Aut}(G),
$$

it is easy to see that $\tilde{\alpha}$ becomes an automorphism of the group algebra $L^{1}(G)$. Denote by $\lambda$ the left regular representation of $G$ as well as the left regular representation of $L^{1}(G)$ on $L^{2}(G)$. Viewing $\tilde{\alpha}, \alpha \in \operatorname{Aut}(G)$, as an automorphism of $\lambda\left(L^{1}(G)\right)$, we show that $\tilde{\alpha}$ can be extended to an automorphism of the von Neumann algebra of the left regular representation, $\mathscr{R}(G)=\lambda\left(L^{1}(G)\right)^{\prime \prime}=\lambda(G)^{\prime \prime}$. We define a unitary operator $U^{\alpha}, \alpha \in \operatorname{Aut}(G)$, by

$$
U^{\alpha} g=\Delta(\alpha)^{-1 / 2} g \circ \alpha^{-1}, \quad g \in L^{2}(G) .
$$

A straight forward calculation shows

$$
\lambda(\widetilde{\alpha}(f))=U^{\alpha} \lambda(f) U^{\alpha^{-1}} .
$$

The unitary implementation $\alpha \mapsto U^{\alpha}$ allows us to define $\widetilde{\alpha}(T)$ for $T \epsilon$ $\mathscr{R}(G)$ by

$$
\tilde{\alpha}(T)=U^{\alpha} T U^{\alpha^{-1}} \text {. }
$$

LEMMA 1.3. The map $\alpha \in \operatorname{Aut}(G) \mapsto U^{\alpha} g \in L^{2}(G)$ is continuous $\left(g \in L^{2}(G)\right)$.

Proof. This follows from Proposition 2, page 78 of [1].

1.4. Our next aim is to study $\operatorname{Aut}(G)$ by embedding it in Aut $\mathscr{R}(G)$, and we shall prove that the embedding is topological if 
Aut $\mathscr{R}(G)$ is provided with the appropriate topology, namely the uniform-weak topology. A neighborhood base at the identity $c \in$ Aut $\mathscr{R}(G)$ is given by

$$
\left\{\alpha \in \text { Aut } \mathscr{R}(G):\left|<(\alpha-\iota) \mathscr{R}_{1}, \phi_{i}>\right|<\varepsilon, \phi_{i} \in \mathscr{R}(G)_{*}, 1 \leqq i \leqq n\right\},
$$

where $\varepsilon>0$ and $\mathscr{R}_{1}$ denotes the unit ball in $\mathscr{R}(G)$. Recall that the predual, $\mathscr{R}(G)_{*}$, is the Fourier algebra $A(G)$ (see [5]). Let

$$
W_{\phi_{1 . .} \phi_{n} ; \varepsilon}=\left\{\alpha \in \operatorname{Aut}(G):\left\|\phi_{i}-\phi_{i} \circ \alpha\right\|<\varepsilon, 1 \leqq i \leqq n\right\}, \quad \phi_{i} \in A(G),
$$

where $\|\cdot\|$ denotes the norm in $A(G)$.

LEMMA 1.5 .

$$
W_{\phi_{1}, \ldots, \phi_{n}: \varepsilon}=\left\{\alpha \in \operatorname{Aut}(G):\left|<(\tilde{\alpha}-\iota) \mathscr{R}_{1}, \phi_{i}>\right|<\varepsilon, \quad 1 \leqq i \leqq n .\right.
$$

Proof. First note $\langle\widetilde{\alpha}(T), \phi\rangle=\langle T, \phi \circ \alpha\rangle$ for $T \in \mathscr{R}(G), \phi \in A(G)$ and $\alpha \in \operatorname{Aut}(G)$; i.e., $\tilde{\alpha}^{t}(\phi)=\phi \circ \alpha$ : If $T=\lambda(f), f \in L^{1}(G)$, we have

$$
\langle\tilde{\alpha}(\lambda(f)), \phi\rangle=\Delta(\alpha)^{-1} \int_{G} f \circ \alpha^{-1}(x) \phi(x) d x=\langle\lambda(f), \phi \circ \alpha\rangle .
$$

Since $\left\{\lambda(f): f \in L^{1}(G)\right\}$ is dense in $\mathscr{R}(G)$, the claim follows. Now $\langle(\widetilde{\alpha}-\iota) T, \phi\rangle=\langle T, \phi \circ \alpha-\phi\rangle, T \in \mathscr{R}_{1}$. Taking the supremum over all $T \in \mathscr{R}_{1}$ we get

$$
\sup _{T \in \mathscr{\mathscr { R } _ { 1 }}}\langle(\tilde{\alpha}-\iota) T, \phi\rangle=\|\phi \circ \alpha-\alpha\|, \quad \phi \in A(G),
$$

and the lemma follows.

Proposition 1.6. The sets $W_{\phi_{1}, \ldots, \phi_{n} ; \epsilon}, \phi_{i} \in A(G)$ and $\varepsilon>0$, form $a$ base at the identity $\iota \in \operatorname{Aut}(G)$ for the Birkhoff topology. Hence the embedding Aut $(G) \hookrightarrow$ Aut $\mathscr{R}(G)$ is topological.

Proof. We show first that the topology generated by the sets $W_{\phi_{1}, \ldots, \phi_{n} ; \varepsilon}$ is weaker then that of $\operatorname{Aut}(G)$. The proof of Lemma 1.5 shows that for $\phi \in A(G), \alpha \in \operatorname{Aut}(G)$.

$$
\|\phi-\phi \circ \alpha\|=\sup _{T \in \mathscr{R}_{1}}|\langle T-\tilde{\alpha}(T), \phi\rangle| .
$$

Writing $\phi=(f \circ \widetilde{g})^{\smile}, f, g \in L^{2}(G)$, we have

$$
\begin{aligned}
\|\phi-\phi \circ \alpha\|= & \sup _{T \in \mathscr{R}_{1}}|\langle(T-\widetilde{a}(T)) f, g\rangle| \\
= & \sup _{T \in \mathscr{R}_{1}}\left|\left\langle\left(T-U^{\alpha} T U^{\alpha-1}\right) f, g\right\rangle\right| \\
= & \sup _{T \in \mathscr{R}_{1}}\left|\left\langle\left(U^{\alpha-1} T-T U^{\alpha-1}\right) f, U^{\alpha^{-1}} g\right\rangle\right| \\
\leqq & \sup _{T \in \mathscr{R}_{1}}\left|\left\langle\left(U^{\alpha-1} T-T\right) f, U^{\alpha-1} g\right\rangle\right| \\
& +\sup _{T \in \mathscr{R}_{1}}\left|\left\langle\left(T-U^{\alpha-1}\right) f, U^{\alpha-1} g\right\rangle\right| .
\end{aligned}
$$


Now

$$
\begin{aligned}
& \left|\left\langle\left(T-T U^{\alpha^{-1}}\right) f, U^{\alpha^{-1}} g\right\rangle\right| \leqq\left\|T\left(f-U^{\alpha-1} f\right)\right\|_{2}\left\|U^{\alpha^{-1}} g\right\|_{2} \\
& \quad \leqq\left\|f-U^{\alpha^{-1}} f\right\|_{2}\|g\|_{2}, \quad \text { all } T \in \mathscr{R}_{1} . \\
& \left|\left\langle\left(U^{\alpha^{-1}} T-T\right) f, U^{\alpha^{-1}} g\right\rangle\right| \\
& \quad=\left|\left\langle U^{\alpha^{-1}} T f, U^{\alpha^{-1}} g\right\rangle-\left\langle T f, U^{\alpha^{-1}} g\right\rangle\right| \\
& \quad=\left|\langle T f, g\rangle-\left\langle T f, U^{\alpha-1} g\right\rangle\right|=\left|\left\langle T f, g-U^{\alpha^{-1}} g\right\rangle\right| \\
& \leqq\|T f\|_{2}\left\|g-U^{\alpha^{-1}} g\right\|_{2} \leqq\|f\|_{2}\left\|g-U^{\alpha^{-1}} g\right\|_{2}, \\
& \quad \text { all } T \in \mathscr{R}_{1} .
\end{aligned}
$$

Let $N$ be a neighborhood of $\iota \in \operatorname{Aut}(G)$ such that $\left\|f-U^{\alpha^{-1}} f\right\|_{2}\|g\|_{2}<$ $\varepsilon / 2$ and $\|f\|_{2}\left\|g-U^{\alpha-1} g\right\|_{2}<\varepsilon / 2$. Then $\|\dot{\phi}-\dot{\phi} \circ \alpha\|<\varepsilon$.

Conversely, let $F \subset G$ be compact and $W$ a neighborhood of $e$ in $G$. Let $U$ be a compact neighborhood of $e$ such that $U^{2} \cdot U^{-1} \subset W$.

Since $A(G)$ is a regular algebra, there exists $\psi \in A(G)$ with $0 \leqq$ $\psi \leqq 1, \psi(u)=1$ for $u \in U$, and support $(\psi) \subset U^{2}$ [5; Lemma 3.2]. Let $\left\{x_{1}, \cdots, x_{n}\right\} \subset F$ be so that $\left\{U x_{i}: 1 \leqq i \leqq n\right\}$ covers $F$. Define $\psi_{i}(y)=$ $\psi\left(y x_{i}^{-1}\right), 1 \leqq i \leqq n$. We claim $W_{\psi_{1}, \ldots, \psi_{n} ; 1} \subset N(F, W)$. Indeed, suppose $\tau \in W_{\psi_{1}, \ldots, \psi_{n} ; 1}$ and let $x \in F$. Then $x \in U x_{j}$ for some $j$. Now $\left\|\psi_{j} \circ \tau-\psi_{j}\right\|<1$ implies $\left\|\psi_{j} \circ \tau-\psi_{j}\right\|_{\infty}<1$, so that $\left|\psi_{j} \circ \tau(x)-\psi_{j}(x)\right|<1$. But for $x \in U x_{j}, \psi_{j}(x)=\psi\left(x x_{j}^{-1}\right)=1$. Hence $\tau(x) \in \operatorname{support}\left(\psi_{j}\right)$, or $\tau(x) \in U^{2} x_{j}$. But then

$$
\tau(x) x^{-1} \in U^{2} x_{j} x^{-1} \in U^{2} U^{-1} \subset W .
$$

In addition

$$
\left\|\psi_{j} \circ \tau^{-1}-\psi_{j}\right\|_{\infty}=\left\|\psi_{j} \circ \tau-\psi_{j}\right\|_{\infty}<1,
$$

so the same argument as above yields $\tau^{-1}(x) \in W x$.

Corollary 1.7. Suppose $G$ has small neighborhoods of the identity, invariant under inner automorphisms (i.e., $G \in[\mathrm{SIN}])$. Then viewing the group Int $(G)$ as a subgroup of Aut $\mathscr{R}(G)$, the pointwise-weak and uniform-weak topologies coincide on Int $(G)$.

Proof. $G \in[\mathrm{SIN}]$ if and only if $\mathscr{R}(G)$ is a finite von Neumann algebra, [4; 13. 10.5]. The conclusion follows from [10; Proposition 3.7].

Note that the above can just as well be stated for [SIN $]_{B}$-groups where $B \subset$ Aut $(G)$ is a subgroup. Also, the corollary is not too surprising in view of the fact that for [SIN]-groups the point-open and Birkhoff topologies of Aut $(G)$ agree on $\operatorname{Int}(G)$ [9; Satz 1.6].

1.8. We say that $G$ is an $[\mathrm{FIA}]_{B}^{-}$-group if $B$ is a relatively 
compact subgroup of Aut $(G)$ (see [7]). It is now a trivial consequence of 1.6 that $G \in[\mathrm{FIA}]_{B}^{-}$if and only if $B$, viewed as a subgroup of Aut $\mathscr{R}(G)$ endowed with the uniform-weak topology, is relatively compact. Cf. [6; Theorem 2.4]. By [6; Corollary 1.6], the pointwiseweak topology may be substituted for the uniform-weak topology.

We mention another consequence of Proposition 1.6 which was suggested to us by Kenneth Ross. An important tool in harmonic analysis on [FIA] $]_{B}^{-}$-groups is the "sharp operator," which is defined as follows: if $f$ is a continuous function on $G \in[\mathrm{FIA}]_{B}^{-}$, then

$$
f^{\sharp}(x)=\int_{B^{-}} f \circ \beta(x) d \beta,
$$

where $d \beta$ is normalized Haar measure on the compact group $B^{-} \subset$ Aut $(G) . \quad f^{\#}$ is a continuous, $B$-invariant function on $G$. We show that if $f$ is in the Fourier algebra $A(G)$, so is $f^{\sharp}$. By Proposition 1.6 the map $\beta \rightarrow f \circ \beta$, Aut $(G) \rightarrow A(G)$, is continuous. Viewing $f^{\sharp}$ as a vector valued integral, we can then adapt [14; Lemma 1.4] to show that $f^{\sharp} \in A(G)$.

1.9. Next we show in an elementary way that for an arbitrary locally compact group $G$, Aut $(G)$ is a complete topological group (in its two-sided uniformity).

TheOREM. Let $G$ be a locally compact group; then Aut $(G)$ is complete with respect to its two-sided uniformity.

Proof. Let $\left(\alpha_{\nu}\right)$ be a Cauchy net in Aut $(G)$. Since $\alpha \mapsto U^{\alpha}$, Aut $(G) \rightarrow \mathscr{L}\left(L^{2}(G)\right)$ is continuous in the strong operator topology, it is also weakly continuous. Now $U^{\alpha} \in \mathscr{L}\left(L^{2}(G)\right)_{1}(=$ unit ball of $\left.\mathscr{L}\left(L^{2}(G)\right)\right)$; also the weak and ultraweak topology coincide on $\mathscr{L}\left(L^{2}(G)\right)_{1}$ and $\mathscr{L}\left(L^{2}(G)\right)_{1}$ is compact in this topology. Thus $\left(U^{\alpha_{\nu}}\right)$ has a point of accumulation $U \in \mathscr{L}\left(L^{2}(G)\right)_{1}$; let $\left(\alpha_{\mu}\right)$ be a subnet such that $U^{\alpha \mu} \underset{\mu}{\longrightarrow} U$ weakly. Then for $f, g \in L^{2}(G)$

$$
\begin{aligned}
& \left\langle\left(U^{\alpha_{\nu}}-U\right) f, g\right\rangle=\left\langle\left(U^{\alpha_{\nu}}-U^{\alpha_{\mu}}\right) f, g\right\rangle+\left\langle\left(U^{\alpha_{\mu}}-U\right) f, g\right\rangle \\
& \quad=\left\langle f-U^{\alpha_{\nu}^{-1} \alpha_{\mu}} f, U^{\alpha_{\nu}^{-1}} g\right\rangle+\left\langle\left(U^{\alpha_{\mu}}-U\right) f, g\right\rangle \\
& \quad \leqq\left\|f-U^{\alpha_{\nu}^{-1} \alpha_{\mu}} f\right\|_{2}\|g\|_{2}+\left\langle\left(U^{\alpha_{\mu}}-U\right) f, g\right\rangle \underset{\mu, \nu}{\longrightarrow} 0
\end{aligned}
$$

since $\alpha_{\nu}^{-1} \alpha_{\mu} \underset{(\nu, \mu)}{\longrightarrow}$ in $\operatorname{Aut}(G)$. Thus $U^{\alpha_{\nu}} \underset{\nu}{ } U$ in the weak operator topology. Similarly $U^{\alpha_{\nu}^{-1}}$ converges weakly to some $V \in \mathscr{L}\left(L^{2}(G)\right)_{1}$. We claim $V=U^{-1}$. Let $f, g \in L^{2}(G), \varepsilon>0$. Let $\nu_{0}$ be such that for $\nu>\nu_{0}$ 


$$
\left|\left\langle U^{\alpha_{\nu}} V f-U V f, g\right\rangle\right|<\varepsilon \text {, and } \| U^{\alpha_{\nu}^{-1}} g-U^{\alpha_{\nu_{0}}^{-1} g \|_{2}}<\frac{\varepsilon}{2\|f\|_{2}} .
$$

Choose $\nu_{1}$ such that $\nu>\nu_{1}$ implies

$$
\left|\left\langle U^{\alpha_{\nu}^{-1}} f-V f, U_{\nu_{0}}^{\alpha_{\nu}^{-1}} g\right\rangle\right|<\varepsilon .
$$

Then for $\nu, \mu>\nu_{0}$ and $\nu_{1}$, we have

$$
\begin{aligned}
& \left|\left\langle U^{\alpha_{\mu}} U^{\alpha_{\nu}^{-1}} f-U V f, g\right\rangle\right| \\
& \quad \leqq\left|\left\langle U^{\alpha_{\mu}} U^{\alpha_{\nu}^{-1}} f-U^{\alpha_{\mu}} V f, g\right\rangle\right|+\left|\left\langle U^{\alpha_{u}} V f-U V f, g\right\rangle\right|,
\end{aligned}
$$

where $\left|\left\langle U^{\alpha_{\mu}} V f-U V f, g\right\rangle\right|<\varepsilon$. Also

$$
\begin{aligned}
\mid & \left\langle U^{\alpha_{\mu}} U_{\nu-1}^{\alpha} f-U^{\alpha \mu} V f, g\right\rangle|=|\left\langle U_{\nu}^{\alpha-1} f-V f, U_{\mu}^{\alpha-1} g\right\rangle \mid \\
& \leqq\left|\left\langle U^{\alpha_{\nu}^{-1}} f-V f, U^{\alpha_{\nu}} g\right\rangle\right|+\left|\left\langle U^{\alpha_{\nu}^{-1}} f-V f, U^{\alpha_{\mu}^{-1}} g-U^{\alpha_{\nu_{0}}^{-1}} g\right\rangle\right| \\
& <\varepsilon+\left\|U_{\nu}^{\alpha_{\nu}^{-1}} f-V f\right\|_{2}\left\|U^{\alpha_{\mu}^{-1}} g-U^{\alpha_{\nu}} g\right\|_{2} \\
& <\varepsilon+2\|f\|_{2}\left\|U^{\alpha_{\mu}^{-1}} g-U^{\alpha_{\nu_{0}}^{-1}} g\right\|_{2}<2 \varepsilon,
\end{aligned}
$$

so that

$$
\left|\left\langle U^{\alpha_{\mu}} U^{\alpha_{\nu}^{-1}} f-U V f, g\right\rangle\right|<3 \varepsilon
$$

But

$$
\left\langle U^{\alpha^{\prime \prime}} U^{\alpha_{\nu}^{-1}} f, g\right\rangle=\left\langle U^{\alpha_{\prime \prime} \alpha_{\nu}^{-1}} f, g\right\rangle \underset{(\mu, \nu)}{\longrightarrow}\langle f, g\rangle,
$$

hence

$$
\langle U V f, g\rangle=\langle f, g\rangle, \quad \text { all } f, g \in L^{2}(G) ;
$$

thus $V=U^{-1}$. In addition,

$$
\langle U f, g\rangle=\lim _{\nu}\left\langle U^{\alpha_{\nu}} f, g\right\rangle=\lim _{\nu}\left\langle f, U^{\alpha_{\nu}^{-1}} g\right\rangle=\langle f, V g\rangle,
$$

so $V=U^{*}$, and we have $U^{-1}=U^{*}$, so $U$ is unitary. A standard argument shows $U^{\alpha_{\nu}}$ converges strongly to $U$ :

$$
\begin{aligned}
& \left\|U^{\alpha_{\nu}} f-U f\right\|_{2}^{2}=\left\langle U^{\alpha_{\nu}} f, U^{\alpha_{\nu}} f\right\rangle-\left\langle U f, U^{\alpha_{\nu}} f\right\rangle \\
& \quad-\left\langle U^{\alpha_{\nu}} f, U f\right\rangle+\langle U f, U f\rangle=2\langle f, f\rangle-\left\langle U f, U^{\alpha_{\nu}} f\right\rangle \\
& \quad-\left\langle U^{\alpha_{\nu}} f, U f\right\rangle \underset{\nu}{\longrightarrow} 0 .
\end{aligned}
$$

It remains to show that $\lambda(x) \mapsto U \lambda(x) U^{-1}$ defines an automorphism of $\lambda(G)$ (and thus of $G$ ). Fix $x \in G$; clearly $\left(\alpha_{\nu}(x)\right)$ is a Cauchy net in $G$ and (since $G$ is complete) converges to an element, say $\alpha(x) \in G$. Then 


$$
U^{\alpha_{\nu}} \lambda(x) U^{\alpha_{\nu}^{-1}}=\lambda\left(\alpha_{\nu}(x)\right) \underset{\nu}{\longrightarrow} \lambda(\alpha(x)) \quad \text { weakly },
$$

and

$$
U^{\alpha_{\nu}} \lambda(x) U^{\alpha_{\nu}^{-1}} \longrightarrow U \lambda(x) U^{-1} \quad \text { weakly } .
$$

So $\lambda(\alpha(x))=U \lambda(x) U^{-1}$. To prove $\alpha$ is a homomorphism,

$$
\begin{aligned}
\lambda(\alpha(x y)) & =U \lambda(x y) U^{-1}=\left(U \lambda(x) U^{-1}\right)\left(U \lambda(y) U^{-1}\right)=\lambda(\alpha(x)) \lambda(\alpha(y)) \\
& =\lambda(\alpha(x) \alpha(y)) ;
\end{aligned}
$$

so $\alpha(x y)=\alpha(x) \alpha(y)$. Also $\lambda\left(\alpha\left(x^{-1}\right)\right)=U \lambda\left(x^{-1}\right) U^{-1}=U \lambda(x)^{-1} U^{-1}=$ $\left(U \lambda(x) U^{-1}\right)^{-1}=\lambda(\alpha(x))^{-1}=\lambda\left(\alpha(x)^{-1}\right)$ i.e., $\alpha\left(x^{-1}\right)=\alpha(x)^{-1}$. To prove continuity of $\alpha$, let $\left(x_{\mu}\right) \rightarrow x_{0}$ in $G$. Then

$$
\lambda\left(\alpha\left(x_{\mu}\right)\right)=U \lambda\left(x_{\mu}\right) U^{-1} \underset{\mu}{\longrightarrow} U \lambda\left(x_{0}\right) U^{-1}=\lambda\left(\alpha\left(x_{0}\right)\right)
$$

in the weak operator topology. But $x \mapsto \lambda(x)$ is a homeomorphism of $G$ onto $\lambda(G)$, where $\lambda(G) \subset \mathscr{L}\left(L^{2}(G)\right)$ carries the weak topology ([6; Lemma 2.2]). Thus $\alpha\left(x_{\mu}\right) \rightarrow \alpha\left(x_{0}\right)$. Similarly, $\alpha^{-1}$ is continuous, and we have $\alpha \in \operatorname{Aut}(G)$, so that $\operatorname{Aut}(G)$ is complete.

REMARK 1.10. Since by 1.6 Aut $(G)$ is topologically embedded in the complete group Aut $\mathscr{R}(G)$, [10; Proposition 3.5], it would be natural to prove completeness of Aut $(G)$ by showing it is closed in Aut $\mathscr{R}(G)$. Actually, such a proof can be given, utilizing the profound duality theory in [16]. We sketch the argument. Consider a net $\left(\alpha_{\nu}\right)$ in $\operatorname{Aut}(G)$ such that $\widetilde{\alpha}_{\nu} \rightarrow \gamma \in \operatorname{Aut} \mathscr{R}(G)$ in the uniform weak topology. By duality theory $\mathscr{R}(G)$ is a Hopf-von Neumann algebra with comultiplication $\delta: \mathscr{R}(G) \rightarrow \mathscr{R}(G) \otimes \mathscr{R}(G)$ which is a $\sigma$-weakly continuous isomorphism given by $\delta(T)=W^{-1}(T \otimes 1) W$, $T \in \mathscr{R}(G)$, where $W k(s, t)=k(s, s t), k \in L^{2}(G \times G), s, t \in G$, [16; Section 4]. Furthermore, one has

$$
\begin{aligned}
\{T \in \mathscr{R}(G): \delta(T)=T \otimes T\} \backslash\{0\} \\
\quad=\{T \in \mathscr{R}(G): T=\lambda(s), \text { for some } s \in G\} .
\end{aligned}
$$

Notice that Aut $(G)$ corresponds to the subgroup

$$
\{\beta \in \text { Aut } \mathscr{R}(G): \delta(\beta \lambda(s))=\beta \lambda(s) \otimes \alpha \lambda(s), \quad \text { all } \quad s \in G\} .
$$

Since $\widetilde{\alpha}_{\nu} \rightarrow \gamma \in \operatorname{Aut}(\mathscr{R}(G))$ and $\delta\left(\widetilde{\alpha}_{\nu} \lambda(s)\right)=\widetilde{\alpha}_{\nu} \lambda(s) \otimes \tilde{\alpha}_{\nu} \lambda(s)$, all $s \in G$, continuity of $\delta$ gives

$$
\delta(\gamma(\lambda(s)))=\gamma(\lambda(s)) \otimes \gamma(\lambda(s)), \quad \text { all } s \in G .
$$

Thus $\gamma=\tilde{\alpha}$ for some $\alpha \in \operatorname{Aut}(G)$. 
CoRollary 1.11. If $G$ is a separable locally compact group, then $\operatorname{Aut}(G)$ is a Polish topological group.

Proof. Indeed, if $G=\bigcup_{n=1}^{\infty} F_{n}, F_{n}$ compact, and if $\left\{U_{m}\right\}_{m \in N}$ is a neighborhood base at $e \in G$, then $\left\{N\left(F_{n}, U_{m}\right)\right\}_{n, m}$ is a neighborhood base at $\iota \in \operatorname{Aut}(G)$, so that $\operatorname{Aut}(G)$ is metrizable $[11 ; 8.3]$ and by 1.9. It is complete.

2. We proceed now to applications of the Theorem in 1.9 First we turn to the question of when certain subgroups of Aut $(G)$ are closed. The following result contains a group theoretical analog to [2; Theorem 3.1]. We thank Erling Stormer for showing us Connes' paper [2], and for helpful discussions concerning central sequences of vov Neumann algebras.

Proposition 2.1. Let $G$ be a separable locally compact group, and $B$ a subgroup of Aut $(G)$. Suppose there is a separable locally compact group $H$ and a continuous surjective homomorphism $\omega: H \rightarrow$ $B$. Then the following are equivalent.

(a) $B$ is closed in Aut $(G)$.

(b) $\omega: H \rightarrow B$ is open onto its range $B$.

(c) For any neighborhood $V$ of the identity in $H$ there exist $\phi_{1}, \cdots, \phi_{n} \in C_{c}(G)$ and $\varepsilon>0$ such that, for all $h \in H$,

$$
\left\|\dot{\phi}_{i} \circ \omega(h)-\dot{\phi}_{i}\right\|_{\infty}<\varepsilon, \quad 1 \leqq i \leqq n, \quad \text { implies } h \in V \cdot(\operatorname{ker} \omega) \text {. }
$$

(d) Same statement as (c) with $C_{c}(G)$ replaced by the Fourier algebra $A(G)$ (and its norm $\|\cdot\|$ ).

Proof. $(a) \Rightarrow(b)$. If $B$ is closed in Aut $(G)$ then $H$ and $B$ are both Polish. Observe then that a continuous homomorphism between two Polish groups is open [12; Corollary 3, p. 98]. (b) $\Rightarrow(\mathrm{c})$. Put $K=\operatorname{ker} \omega$. Since $\omega$ is open it follows from Lemma 1.1. that given a neighborhood $V$ of the identity in $H$ there are functions $\phi_{1}, \cdots, \phi_{n} \in$ $C_{c}(G)$ and $\varepsilon>0$ so that $W_{\phi_{1}, \ldots, \phi_{n} ; \varepsilon} \cap B \subset \omega(V)$. Now $\omega$ can be lifted to a map $\tilde{\omega}$ of $H / K \rightarrow B$, so that the diagram commutes and $\tilde{\omega}$ is a homeomorphism.

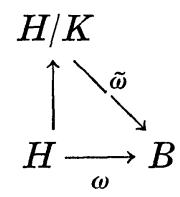

Thus $\omega(h) \in W_{\phi_{1}, \ldots, \phi_{n} ; \epsilon}$ implies $\omega(h) \in \omega(V)=\tilde{\omega}(V K) ;$ hence $\tilde{\omega}(h K) \epsilon$ $\tilde{\omega}(V K)$, so that $h \in h K \subset V K$. 
$(c) \Rightarrow(d)$ is clear in view of Proposition 1.6.

$(\mathrm{d}) \Rightarrow(\mathrm{a})$. By 1.6 and 1.11 there is a sequence $\left(\phi_{n}\right)$ from $A(G)$ such that the sets $W_{n}=W_{\phi_{1}, \ldots, \phi_{n} ; 1 / n}$ form a base for the identity in $\operatorname{Aut}(G)$. Let $\left\{V_{n}\right\}$ be a countable base for the identity in $H$. By (d), given $n$ there is an $m(n)$ so that $\omega(h) \in W_{m(n)}$ implies $h \in V_{n} K$. Let $\theta \in B^{-}$and choose a sequence $\left(\alpha_{n}\right)$ from $B$ so that $\alpha_{n} \rightarrow \theta$ and $\alpha_{n+j}^{-1} \alpha_{n} \in$ $W_{m(n)}$ for $j \geqq 0$. Setting $\tilde{\omega}^{-1}\left(\alpha_{n}\right)=h_{n} K$, we have $h_{n+j}^{-1} h_{n} \cdot K \subset V_{n} K$, $j \geqq 0$. This says that $\left(h_{n} K\right)$ is Cauchy in the left uniformity of $H / K$. Since $H / K$ is locally compact, it is complete, and $h_{n} K \vec{n}$ $h K \in H / K$, hence $\omega(h)=\tilde{\omega}(h K)=\theta$ by continuity of $\tilde{\omega}$, and thus $\theta \in B$.

2.2. Define a homomorphism Ad: $G \rightarrow \operatorname{Int}(G)$ by $\operatorname{Ad}(g)(x)=g x g^{-1}$. A sequence $\left(x_{n}\right)$ from $G$ is said to be central if $\operatorname{Ad}\left(x_{n}\right) \vec{n}_{n}$ : in Aut $(G)$. $\left(x_{n}\right)$ is trivial if there is a sequence $\left(z_{n}\right)$ from the center $Z(G)$ of $G$ such that $x_{n} z_{n}^{-1} \underset{n}{\longrightarrow} e$.

CoROLlary. Let $G$ be separable locally compact. Then Int $(G)$ is closed if and only if all central sequences are trivial.

Proof. If Int $(G)$ is closed, let $\left(x_{n}\right)$ be a central sequence and $\left\{V_{n}\right\}$ a nested neighborhood base for the identity in $G$. By (d) of 2.1 for each $n$ we can find a set $\left\{\phi, \cdots, \phi_{i_{n}}\right\} \subset A(G)$ and $\varepsilon_{n}>0$ so that for $x \in G,\left\|\phi_{j} \circ \operatorname{Ad}(x)-\dot{\phi}_{j}\right\|<\varepsilon_{n}, 1 \leqq j \leqq i_{n}$, implies $x \in V_{n} Z(G)$. Note that if $\omega=\mathrm{Ad}$ in 2.1 , ker $\omega$ is just $Z(G)$. Choosing a sequence $\left(k_{j}\right)$ from $N$ such that $k \geqq k_{j} \Longrightarrow\left\|\dot{\phi}_{j} \circ \mathrm{Ad}\left(x_{k}\right)-\phi_{j}\right\|<\varepsilon_{n}, 1 \leqq j \leqq i_{n}$, we have $x_{k} \in V_{n} Z(G)$, hence $x_{k} z_{k}^{-1} \in V_{n}$ for some $z_{k} \in Z(G)$. Then $x_{k} z_{k}^{-1} \rightarrow e$, and $\left(x_{n}\right)$ is trivial. The converse is shown the same way as $(\mathrm{d}) \Rightarrow(\mathrm{a})$ in 2.1.

2.3. We remark that the class of groups for which Aut $(G)$ is locally compact includes the compactly generated Lie groups [9; Satz 2.2]. For $\operatorname{Int}(G)$ we have the following

Corollary. Let $G$ be separable and locally compact. Then Int $(G)$ is locally compact $\Leftrightarrow \operatorname{Int}(G)$ is closed.

Proof. If Int $(G)$ is locally compact, it is necessarily closed [9; Theorem 5.11]. On the other hand if Int $(G)$ is closed, take $G=H$ and $\omega=\operatorname{Ad}$ in 2.1. Then by continuity of $\operatorname{Ad}, \operatorname{Int}(G)$ is homeomorphic with $G / Z(G)$.

2.4. If Int $(G)$ is not closed it is still reasonable to ask if $\operatorname{Int}(G)^{-}$ will be locally compact. 
COROLlaRY. Let $G$ be a separable, connected locally compact group. Then the closure Int $(G)^{-}$in Aut $(G)$ is locally compact.

Proof. By [17; Lemma 2.2] there is a locally compact connected group $P$ and a continuous map $\rho_{G}: P \rightarrow \operatorname{Aut}(G)$ with $\rho_{G}(P)=\operatorname{Int}(G)^{-}$. Since $G$ is separable, it follows from the construction of $P$ in [17] that $P$ is also separable. Thus by Corollary 1.11 and [12; Corollary 3] $\rho_{G}$ is a homeomorphism and hence Int $(G)^{-}$is locally compact.

We now give an example that shows that for nonconnected groups, Int $(G)^{-}$need not be locally compact. Let $G$ be the countable weak direct sum of the free group on two generators with the discrete topology: $G=\sum_{n=1}^{\infty} G_{n}$, where $G_{n}$ is generated by $\left\{a_{n}, b_{n}\right\}$. The neutral element of $G_{n}$ is the empty word, $\Phi_{n}$, and $e=\left(\Phi_{1}, \Phi_{2}, \cdots\right)$ is the neutral element of $G$. If Int $(G)^{-}$were locally compact there would be a relatively compact open neighborhood $N$ of the identity $c$ in Int $(G)$. If $N_{1}$ is another open neighborhood of $\iota$, since $\bigcup_{x \in G} N_{1}^{-} \operatorname{Ad}(x)$ covers Int $(G)^{-}$, there would be a finite subcover, $N^{-} \subset \bigcup_{i=1}^{n} N_{1}^{-} \operatorname{Ad}\left(x_{i}\right)$ of $\mathrm{N}^{-}$. Thus

$$
N=N^{-} \cap \operatorname{Int}(G) \subset\left[\bigcup_{i=1}^{n} N_{1}^{-} \operatorname{Ad}\left(x_{i}\right)\right] \cap \operatorname{Int}(G)=\bigcup_{i=1}^{n} N_{1} \operatorname{Ad}\left(x_{i}\right) .
$$

We may assume $N=N(C,\{e\})$, where $C=\left\{a_{1}, b_{1}\right\} \times\left\{a_{2}, b_{2}\right\} \times \cdots \times$ $\left\{a_{n}, b_{n}\right\} \times\left\{\Phi_{n+1}\right\} \times \cdots$, since $N$ must contain a neighborhood of this form. It is then easy to see $\mathrm{Ad}(g) \in N$ if and only if $g=\left(\Phi_{1}, \Phi_{2}, \cdots \Phi_{n}\right.$, $\left.g_{n+1}, \cdots\right), g_{n+j} \in G_{n+j}, j \geqq 1$. Let $N_{1}=N\left(C^{\prime},\{e\}\right), C^{\prime}=\left\{a_{1}, b_{1}\right\} \times \cdots \times$ $\left\{a_{n+1}, b_{n+1}\right\} \times\left\{\Phi_{n+2}\right\} \times \cdots$. Then $N$ and $N_{1}$ are subgroups, $\operatorname{Ad}(g) \in N_{1}$ iff $g=\left(\Phi_{1}, \cdots, \Phi_{n}, \Phi_{n+1}, g_{n+2}, \cdots\right) g_{n+j} \in G_{n+j}, j \geqq 2 . \quad N_{1}$ is normal in $N$ and $N / N_{1} \cong G_{n+1}$. This contradicts (*).

2.5. Let $G_{F}$ be the closed normal subgroup of elements $x$ in $G$ having relatively compact conjugacy classes $\left\{g x g^{-1}: g \in G\right\}$. If $G \in$ [SIN], $G_{F}$ is open since any compact Int $(G)$-invariant neighborhood of $e$ is contained in $G_{F}$. Let $\omega: G \rightarrow \operatorname{Aut}\left(G_{F}\right)$ be the continuous homomorphism $\omega(g)=\left.\operatorname{Ad}(g)\right|_{G_{F}}$, and let $B$ be the subgroup $\omega(G) \subset \operatorname{Aut}\left(G_{F}\right)$. Clearly $G_{F}$ is an $[\mathrm{SIN}]_{B}$-group, and we have

CoRollary. Let $G$ be separable. Then, with notation as above, $B$ is closed $\Leftrightarrow B$ is compact $\Leftrightarrow G / \operatorname{ker} \omega$ is compact.

Proof. The first equivalence is proved in [7]. If $B$ is closed, $B$ is homomorphic with $G / \operatorname{ker} \omega$ (the proposition in 2.1 , (a) $\Rightarrow(b)$ ) so by compactness of $B, G / \operatorname{ker} \omega$ must be compact. Conversely, if $G / \operatorname{ker} \omega$ is compact then so is $B=\tilde{\omega}(G / \operatorname{ker} \omega)$ by continuity of the lifted map $\tilde{\omega}$. 
Specializing the preceding corollary even further we obtain

COROLLARY 2.6. Let $G$ be a locally compact group and suppose Int $(G)^{-}$is compact. Then Int $(G)$ is closed $\Leftrightarrow G / Z(G)$ is compact $(Z(G)=$ the center of $(G))$.

Proof. This follows immediately from the Corollary in 2.5 if $G$ is separable. From [7] Int $(G)$ is closed $\Leftrightarrow \operatorname{Int}(G)$ is compact. But Int $(G)$ compact implies Ad: $G \rightarrow \operatorname{Int}(G)$ is open [11; Theorem 5.29], hence $\operatorname{Int}(G) \cong G / Z(G)$, and so $G / Z(G)$ is compact. Conversely if $G / Z(G)$ is compact, lifting Ad to a continuous map $G / Z(G) \rightarrow \operatorname{Int}(G)$ we see that $\operatorname{Int}(G)$ is compact, hence closed.

CoROLlaRY 2.7. Let $G$ be a separable locally compact group. Then Int $(G)$ is unimodular $\Leftrightarrow G$ is unimodular and Int $(G)$ is closed.

Proof. If Int $(G)$ is unimodular, in particular it is closed, so by the proposition in 2.1 it is topologically isomorphic with $G / Z(G)$, so that the latter is unimodular. It is then easy to see $G$ is unimodular; we give a proof for completeness. Let $d z$ and $d \dot{x}$ be Haar measures on $Z(G)$ and $G / Z(G)$ respectively, and $x \mapsto \dot{x}, G \mapsto G / Z(G)$ the canonical map. Let

$$
\mu(\phi)=\int_{G / Z(G)} \int_{Z /(G)} \phi(x z) d z d \dot{x}, \quad \phi \in C_{c}(G) .
$$

By the Weil integration formula $\mu$ is a left Haar measure on $G$. Using right-invariance of $d \dot{x}$ and the fact that $Z(G)$ is the center, one verifies easily that $\mu$ is even right-invariant. Thus $G$ is unimodular. Conversely, if $G$ is unimodular and $\operatorname{Int}(G)$ is closed we show that $G / Z(G)$ is unimodular. It will then follow that $\operatorname{Int}(G)$ is unimodular, since $\operatorname{Int}(G) \cong G / Z(G)$.

Define $\mu$ as above. By assumption $\mu$ is right-invariant. The mapping $C_{c}(G) \rightarrow C_{c}(G / Z(G)), \phi \mapsto \tilde{\phi}, \tilde{\phi}(\dot{x})=\int_{z(G)} \phi(x z) d z$ is surjective [11, Theorem 15.21]. $\mu(\phi)=\mu\left(\phi_{y}\right)$ for all $\phi \in C_{c}(G), y \in G$, then implies $d \dot{x}$ is right-invariant:

$$
\int_{G / Z(G)} \tilde{\phi}_{\dot{y}}(\dot{x}) d \dot{x}=\mu\left(\phi_{\dot{y}}\right)=\mu(\phi)=\int_{G / Z(G)} \tilde{\phi}(\dot{x}) d \dot{x}
$$

(here $\phi_{y}(x)=\phi(y x)$ ). Thus Int $(G)$ is unimodular.

Finally we show that closedness of Int $(G)$ does not imply closedness of Int $\mathscr{R}(G)$, nor conversely. 
Proposition 2.8. There is a group $G$ such that Int $(G)$ is closed and Int $\mathscr{R}(G)$ is nonclosed. On the other hand, there is a group $G$ with Int $(G)$ nonclosed and Int $\mathscr{R}(G)$ closed.

Before proving the proposition we need a fact, the proof of which we include for the sake of completeness. If $Q$ and $Q^{*}$ represent the rationals and nonzero rationals respectively, let $G=\left\{(p, q): p \in Q^{*}, q \in Q\right\}$ with multiplication $(p, q)\left(p^{\prime}, q^{\prime}\right)=\left(p p^{\prime}, q+p q^{\prime}\right)$. Provide $G$ with the discrete topology. Then Aut $(G)=\operatorname{Int}(G)$. To see this, let $\alpha \in \operatorname{Aut}(G)$ and set $\alpha(1, q)=\left(\alpha_{1}(q), \alpha_{2}(q)\right), q \in Q$. Now $\alpha(1, q) \alpha\left(1, q^{\prime}\right)=\left(\alpha_{1}(q) \alpha_{1}\left(q^{\prime}\right)\right.$, $\left.\alpha_{2}(q)+\alpha_{1}(q) \alpha_{2}\left(q^{\prime}\right)\right)$. Also, $\alpha\left[(1, q)\left(1, q^{\prime}\right)\right]=\left(\alpha_{1}\left(q+q^{\prime}\right), \alpha_{2}\left(q+q^{\prime}\right)\right)$. This forces $\alpha_{1}\left(q+q^{\prime}\right)=\alpha_{1}(q) \alpha_{1}\left(q^{\prime}\right)$ and thus $\alpha_{1}(q)=1$ for all $q \in Q$, since the only homomorphism of the additive group $(Q,+)$ into the multiplicative group $\left(Q^{*}, \cdot\right)$ is the trivial one. Thus $\alpha_{2}\left(q+q^{\prime}\right)=\alpha_{2}(q)+$ $\alpha_{2}\left(q^{\prime}\right)$, so $\alpha_{2} \in \operatorname{Aut}(Q,+)$, and so $\alpha_{2}(q)=a q, \alpha \in Q^{*}$. Set $\alpha(q, 0)=$ $\left(\beta_{1}(p), \beta_{2}(p)\right), p \in Q^{*}$. We calculate $\alpha(p, q)=\alpha[(p, 0)(1, q / p)]=$ $\alpha(p, 0) \alpha(1, q / p)=\left(\beta_{1}(p), \beta_{2}(p)+\beta_{1}(p) \cdot(\alpha q / p)\right)$. But also

$$
\begin{aligned}
\alpha(p, q) & =\alpha[(1, q)(p, 0)]=\alpha(1, q) \alpha(p, 0) \\
& =\left(\beta_{1}(p), \alpha q+\beta_{2}(p)\right) .
\end{aligned}
$$

We have $\beta_{2}(p)+(a q / p) \beta_{1}(p)=a q+\beta_{2}(p)$, and hence $\beta_{1}(p)=p$. Furthermore, equating $\alpha(p, 0) \alpha\left(p^{\prime}, 0\right)$ with $\alpha\left(p^{\prime}, 0\right) \alpha(p, 0),\left(p, p^{\prime} \in Q^{*}\right)$, we arrive at $\beta_{2}(p)\left(1-p^{\prime}\right)=\beta_{2}\left(p^{\prime}\right)(1-p)$. If $p, p^{\prime} \neq 1$, then $\beta_{2}(p) /(1-p)=$ $\beta_{2}\left(p^{\prime}\right) /\left(1-p^{\prime}\right)=b \in Q$, a constant. Thus $\beta_{2}(p)=b(1-p), p \neq 1, p \in Q^{*}$. But since $\alpha(1,0)=(1,0), \beta_{2}(1)=0$, so the equation holds for all $p \in Q^{*}$. Now $\alpha$ has been completely determined:

$$
\begin{aligned}
\alpha(p, q) & =\alpha[(1, q)(p, 0)(p, 0)] \\
& =(p, \alpha q+b(1-p)) .
\end{aligned}
$$

But $(a, b)(p, q)(a, b)^{-1}=(p, a q+b(1-p))$, which means $\alpha \in \operatorname{Int}(G)$.

Proof of Proposition 2.8. Let $G$ be the group described above. Since all the nontrivial conjugacy classes of $G$ are infinite, $\mathscr{R}(G)$ is a type $\Pi_{3}$ factor. Since $G$ is amenable, $\mathscr{R}(G)$ must be the hyperfinite factor [3; Corollary 7.2], hence Int $\mathscr{R}(G)$ is nonclosed.

For the other direction, let $A=\left(\prod_{1}^{\infty} Z_{2}\right) \oplus\left(\sum_{1}^{\infty} Z_{2}\right)$, where $\prod_{1}^{\infty} Z_{2}$ has the product topology and the weak direct sum $\sum_{1}^{\infty} Z_{2}$ the discrete topology. Define $\alpha: A \rightarrow A$ as follows

$$
\alpha\left(\left(z_{i}\right),\left(w_{i}\right)\right)=\left(\left(z_{i}+w_{i}\right),\left(w_{i}\right)\right),\left(z_{i}\right) \in \prod_{1}^{\infty} Z_{2},\left(w_{i}\right) \in \sum_{1}^{\infty} Z_{2} .
$$

Then $\alpha$ is a continuous homomorphism and $\alpha^{2}=$ identity, so that $\alpha \in \operatorname{Aut}(A)$. Let $G$ be the semidirect product $G=A x_{\eta} Z_{2}$, where 
$\eta(m)=\alpha^{m}, m \in Z_{2}{ }^{*}$. Since $\alpha$ leaves the elements of $\sum_{1}^{\infty} Z_{2}$ fixed, it follows that $G / \Pi_{1}^{\infty} Z_{2}$ is abelian so that the commutator $[G, G]$ is compact. In particular all the conjugacy classes of $G$ are precompact. Furthermore one sees that the center $Z(G)$ is equal to $\Pi_{1}^{\infty} Z_{2}$ so $G / Z(G)$ is noncompact. Since $Z /(G)$ is open it is clear that $G$ has small invariant neighborhoods of the identity, and by the Ascoli theorem for groups [7; Satz 1.7], Int $(G)^{-}$is compact. According to Corollary 2.6, Int $(G)$ is not closed in Aut $(G)$. This can also be seen directly: let $\tau\left(\left(x_{i}\right),\left(y_{i}\right), 0\right)=\left(\left(x_{i}\right),\left(y_{i}\right), 0\right)$ and $\tau\left(\left(x_{i}\right),\left(y_{i}\right), 1\right)=$ $\left(\left(x_{i}+1\right),\left(y_{2}\right), 0\right)$, where $\left(x_{i}\right) \in \prod_{1}^{\infty} Z_{2},\left(y_{i}\right) \in \sum_{1}^{\infty} Z_{2}$.

Then

$$
\tau \in \operatorname{Int}(G)^{-} \backslash \operatorname{Int}(G)
$$

Observe next that $G$ is type $I$, containing a normal abelian subgroup $A$ of finite index, thus Int $\mathscr{R}(G)=\{\alpha \in$ Aut $\mathscr{R}(G): \alpha$ leaves the center of $\mathscr{R}(G)$ pointwise fixed $\}$ is closed [15; Corollary 2.9. 32].

\section{REFERENCES}

1. J. Broconnier, Sur les groupes topologiques localment compact, J. Math. Pure Appl., 27 (1948), 1-85.

2. A. Connes, Almost periodic states and factors of type $I I I_{1}$, J. Funct. Anal., 16 (1974), 415-445.

3. Classification of injective factors, Ann. Math., 104 (1976), 73-115.

4. J. Dixmier, Les $C^{*}$-Algèbres et Leurs Représentations, Gauthier-Villars, 1964.

5. P. Eymard, L'algèbre de Jourier d'un groupe localment compact, Bull. Soc. Math. France, 92 (1964), 181-236.

6. W. Green, Compact groups of automorphisms of von Neumann algebras, Math. Scand., 37 (1975), 284-295.

7. S. Grosser and M. Moskowitz, Compactness conditions in topological groups, J. Reine Angew. Math., 246 (1971), 1-40.

8. - On central topological groups, Trans. Amer. Math. Soc., 127 (1967), 317340.

9. S. Grosser, O. Loos, and M. Moskowitz, Ueber Automorphismengrouppen LokalKompakter Gruppen und Derivationen von Lie-Gruppen, Math. Z., 114 (1970), 321-339. 10. U. Haagerup, The standard form of von Neumann algebras, Math. Scand., 37 (1975), 271-283.

11. E. Hewitt and K. Ross, Abstract Harmonic Analysis, I, Springer-Verlag, 1963.

12. T. Husain, Introduction to Topological Groups, W. B. Saunders, 1966.

13. J. Liukkonen, Dual spaces of groups with precompact conjugacy classes, Trans. Amer. Math. Soc., 180 (1973), 85-108.

14. R. Mosak, The $L^{1}$ - and $C^{*}$-algebras of $[F I A]_{B}^{-}$groups and their representations, Trans, Amer. Math. Soc., 163 (1972), 277-310.

15. S. Sakai, $C^{*}$-Algebras and $W^{*}$-Algebras, Springer-Verlag, 1971.

16. M. Takesaki and N. Tatsuuma, Duality and subgroups, Ann. Math., 93 (1971), 344-364.

17. D. Zerling, (CA) topological groups, Proc. Amer. Math. Soc., 54 (1976), 345-351.

* This example has appeared in $[13 ;$ p. 104]. 
Received April 29, 1977 and in revised form September 30, 1977. The results of this paper were presented by the first-named author to the American Mathematical Society at the Seattle meeting on August 15, 1977.

Iowa State UnIVERSITy

AMES, IA 50011

AND

UNIVERSITY OF OSLO

OSLO, NORWAY 


\section{PACIFIC JOURNAL OF MATHEMATICS}

\section{EDITORS}

RICHARD ARENS (Managing Editor)

University of California

Los Angeles, California 90024

C. W. CURTIS

University of Oregon

Eugene, OR 97403

C. C. MOORE

University of California

Berkeley, CA 94720

\section{J. DUGUNDJI}

Department of Mathematics University of Southern California Los Angeles, California 90007

R. Finn aNd J. Milgram Stanford University Stanford, California 94305

\section{ASSOCIATE EDITORS}

E. F. BeCKenbaCH

B. H. NeumanN

F. WOLF

K. YosHIDA

\section{SUPPORTING INSTITUTIONS}

UNIVERSITY OF BRITISH COLUMBIA CALIFORNIA INSTITUTE OF TECHNOLOGY UNIVERSITY OF CALIFORNIA MONTANA STATE UNIVERSITY UNIVERSITY OF NEVADA, RENO NEW MEXICO STATE UNIVERSITY OREGON STATE UNIVERSITY UNIVERSITY OF OREGON
UNIVERSITY OF SOUTHERN CALIFORNIA STANFORD UNIVERSITY UNIVERSITY OF HAWAII UNIVERSITY OF TOKYO UNIVERSITY OF UTAH WASHINGTON STATE UNIVERSITY UNIVERSITY OF WASHINGTON 


\section{Pacific Journal of Mathematics}

\section{Vol. 76, No. $1 \quad$ November, 1978}

Ata Nuri Al-Hussaini, Potential operators and equimeasurability ......... 1

Tim Anderson and Erwin Kleinfeld, Semisimple nil algebras of type $\delta . \ldots .99$

Stephen LaVern Campbell, Linear operators for which $T^{*} T$ and $T+T^{*}$

commute. III ......................................

Robert Jay Daverman, Special approximations to embeddings of codimension one spheres...............................

Donald M. Davis, Connective coverings of $\mathrm{BO}$ and immersions of projective

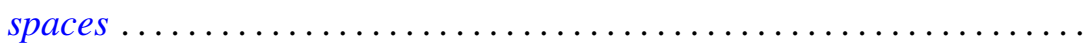

V. L. (Vagn Lundsgaard) Hansen, The homotopy type of the space of maps of

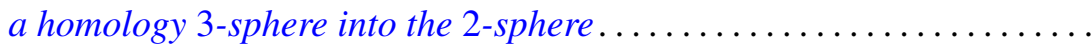

James Victor Herod, A product integral representation for the generalized

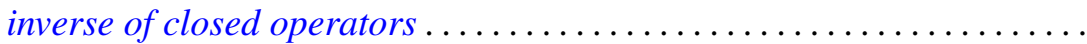

A. A. Iskander, Definability in the lattice of ring varieties ..............

Russell Allan Johnson, Existence of a strong lifting commuting with a

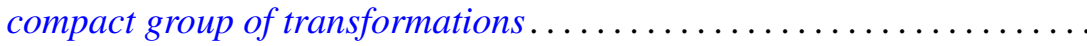

Heikki J. K. Junnila, Neighbornets...................... 83

Klaus Kalb, On the expansion in joint generalized eigenvectors . ......... 109

F. J. Martinelli, Construction of generalized normal numbers . . . . . . . . . 117

Edward O'Neill, On Massey products ....................... 123

Vern Ival Paulsen, Continuous canonical forms for matrices under unitary

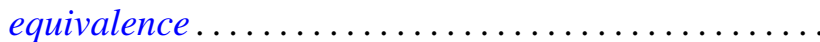

Justin Peters and Terje Sund, Automorphisms of locally compact groups . . . 143

Duane Randall, Tangent frame fields on spin manifolds . . . .

Jeffrey Brian Remmel, Realizing partial orderings by classes of co-simple sets . . . .

J. Hyam Rubinstein, One-sided Heegaard splittings of 3-manifolds ...

Donald Charles Rung, Meier type theorems for general boundary approach

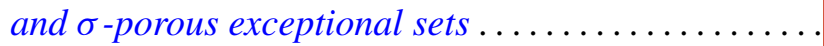

Ryōtarō Satō, Positive operators and the ergodic theorem

Ira H. Shavel, A class of algebraic surfaces of general type constructed from

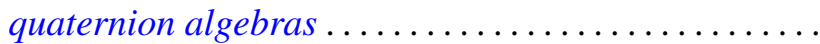

Patrick F. Smith, Decomposing modules into projectives and injectives ....

Sergio Eduardo Zarantonello, The sheaf of outer functions in the polydisc... 